\title{
Review \\ Treatment strategy and efficacy of imaging-guided radiofrequency ablation in large hepatocellular carcinoma
}

Yang Wei Chen Min Hua*

Treatment strategy and efficacy of imaging-guided radiofrequency ablation in large hepatocellular carcinoma

Yang Wei and Chen Min Hua*

\begin{abstract}
Radiofrequency (RF) ablation was recently introduced as a novel ablation modality for hepatocellular carcinoma (HCC). Several investigators have reported that percutaneous RF ablation for patients with small HCC nodules provide favorable survival with excellent local control. However, several limitations of RFA have been pointed out, such as limited coagulated necrosis and relatively frequent local recurrences in large HCC. The critical factors for treatment success in large tumors included (a) the target diameter of an ablation- or of overlapping ablations - must be larger than the diameter of the tumor that undergoes treatment; (b) reduce the negative effect of blood flow and tissue perfusion on RF heating. To overcome RF limitations, researchers developed several techniques and skills of percutaneous treatments and several studies have demonstrated that multiple RF ablation processes with overlapping of ablation zones or combination therapies are feasible for large liver tumors. In this review, we analyzed varies of treatment strategies for percutaneous RF of large tumors and summarized the efficacy of clinical application.
\end{abstract}

Key words: radiofrequency ablation, hepatocellular carcinoma, large tumor, treatment strategy

Accepted on Apr. 1, 2009

Department of Ultrasound, Peking University, Key laboratory of Carcinogenesis and Translational Research (Ministry of Education), Peking University School of Oncology, Beijing Cancer Hospital \& Institute

Address: No 52 Fucheng RD, Beijing 100142, China

* Corresponding author: Chen Min Hua

Address: Ultrasound department, Key laboratory of Carcinogenesis and translational Research (Ministry of Education), Peking University School of Oncology, Beijing Cancer Hospital \& Institute 100142, China

Tel: 8610-88196299 Fax: 8610-88140655

E-mail: minhuachen@bjcancer.org 


\section{Introduction}

RF ablation was recently introduced as a novel ablation modality for $\mathrm{HCC}^{\left.1{ }^{2}\right)}$. For adequate destruction of tumor tissue, the entire target volume must be subjected to cytotoxic temperatures. Different physical mechanisms are involved in the hepatic hyperthermic treatments in order to generate a lethal temperature. A common important factor that affects the success of thermal ablation is the ability to ablate all viable tumor tissue and possibly an adequate tumor-free margin. Ideally, a $360^{\circ}, 0.5-1-\mathrm{cm}$-thick ablative margin should be produced around the tumor. This cuff would ensure that microscopic invasions around the periphery of a tumor have been eradicated. Thus, the target diameter of an ablation- or of overlapping ablations - must be larger than the diameter of the tumor that undergoes treatment ${ }^{3)}{ }^{4)}$. Most technologies enable us to produce liver thermal lesions of approximately $3-3.5 \mathrm{~cm}$ in diameter; RFA has consequently become an emerging percutaneous therapeutic option both for small $\mathrm{HCC}^{5 \text { () }}$. New techniques, strategies (for example, multiple RF ablation processes with overlapping of ablation zones) and combined therapies (tumor ischemia and RFA) have made it possible to treat large tumors. In this review, we analyzed varies of treatment strategies for percutaneous RF of large tumors and summarized the efficacy of clinical application.

\section{Overlapping model and method}

Because most RF device produced limited ablation area and the complete ablation of a tumor requires safety margins, these performances are clearly insufficient for the proper treatment of a tumor target larger than $3 \mathrm{~cm}$ in a single application. Therefore, to ensure entire coverage of the targeted zone with monopolar devices, multiple overlapping RF ablations with successive positionings of the electrode are required. Dodd et $\mathrm{al}^{7)}$ reported their results of computer analysis of the thermal injury sizes created by overlapping ablations and proposed 6 and 14-ablation models. Their results demonstrated the importance of performing these types of calculations to develop tumor ablation strategies. Using a mathematical model to calculate the optimal number of RF ablation process for the target tumor, our group established a preoper- ative protocol for ultrasonographically guided percutaneous $\mathrm{RF}$ ablation of large liver tumors ${ }^{8}$. A regular prism and a regular polyhedron model were used to develop a preoperative protocol for liver tumor ablation (Fig. 1). This protocol enabled the authors to minimize the number of ablation spheres, optimize the overlapping mode, and determine the electrode placement process. The described protocol have been reported a complete tumor ablation rate of $87.6 \%$ in 110 patients with large HCC. The author also mentioned some important factors for electrode placement: (a) precise placement of the electrode with reference to the surrounding anatomic structures during the RF procedure. In large tumors at the surface of the liver or tumors in which there was no marker for reference, adjuvant measures could be used for precise positioning of the RF electrode. For example, 2 or 3 very small needles were inserted into the target sites to serve as markers for the electrode placements. (b) monitoring the actual site of the electrode tip by performing multi-plane scanning from different directions after placing the electrode. (c) Replacement electrodes or adding a number of electrode placements was required when tissue was insufficiently ablated because the electrode tip deviated from the predetermined position.

The role of Contrast ultrasound in RF of large $\mathrm{HCC}$

Recently, further developments of contrast-enhanced US (CEUS) technique have significantly increased its clinical utility. Continuous mode, low MI scans performed with harmonic imaging and contrast specific software appears as a very useful technique for the visualization of both macro- and microcirculation with depiction of tumor vascularisation. Solbiati et al first reported the role of CEUS before, during and immediately at the end of RF ablation procedures to monitor and assess the therapeutic result prior to closing the treatment session ${ }^{9)}$.

Our group reported identification of the exact size and extent of HCC before RFA are important for designing treatment protocols. Large infiltrative $\mathrm{HCC}$ have an indistinct margin and no capsule. US can only depict the approximate shape of the hyperechoic or hypoechoic tumour area, but CEUS provides functional, as well as anatomical, information 


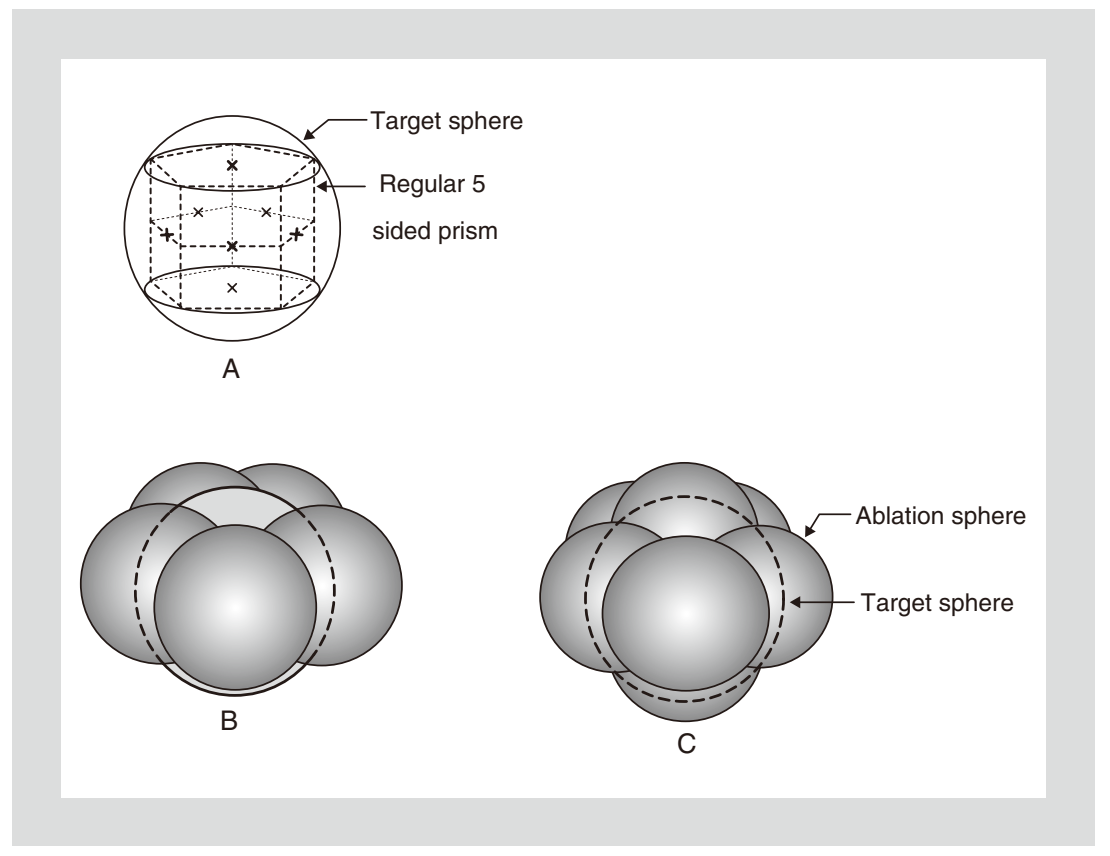

\section{Figure 1 Computer representations of a regular 5 -sided prism model}

A: A regular 5-sided prism is inscribed in a target sphere. The ablation model is constructed by performing 5 ablations on the 5 lateral sides, 1 ablation on the upper side, and another ablation on the lower side, for a total of 7 ablations. B: 5 ablations are performed on lateral sides of the regular prism. C: 2 additional spheres are used to cover the upper and lower sides. The drawing depicts the ablation volume encompassing the target sphere. about blood flow in the tumour, and is especially useful in detecting abnormal blood vessel architecture that surrounds a tumour. Livraghi, et al reported the RFA results of medium and large $\mathrm{HCC}$, and found that there was a distinct difference in tumour necrosis rate between invasive tumours and non-invasive tumours. One reason for this might be that conventional US could not accurately measure the size of invasive tumours before treatment, resulting in insufficient ablation. In our study, of the lesions with indistinct margins on US, $71 \%$ were larger and had better defined margins on CEUS. However, $62 \%$ of the tumours with distinct margins or capsules showed no observed changes in size after contrast agent administration. Thus, it is crucial to define the exact morphological features of HCC, especially in large and invasive tumors, before RFA treatment ${ }^{10)}$.

\section{Strategy to decrease heat sink effect}

Several strategies have been used successfully to reduce the negative effect of blood flow on RF heating. Results of many clinical and experimental studies have shown that decreasing hepatic inflow or outflow by using a mechanical maneuver (balloon, clamping) ${ }^{11}{ }^{12}$ or pharmacologic manipu- lation ${ }^{13)}$ increases the volume and reproducibility of thermal ablation achieved with RF ablation. The main blood supply of $\mathrm{HCC}$ is derived from the artery. Hence, transarterial occlusion of its blood supply before heat ablation may significantly increase the size of the ablation lesion. Buscarini et al $^{14)}$ and Rossi et $a l^{15)}$ have reported the use of RF to ablate large HCCs $(>3.5 \mathrm{~cm})$ after interruption of the tumor's arterial blood supply by segmental embolization or balloon occlusion of the hepatic artery. In a study of 62 patients with HCCs 3.5 to $8.5 \mathrm{~cm}$ treated by this approach, Rossi et al reported no major complications and a $90 \%$ complete response rate. A 1-year survival rate of $87 \%$ was reported, but the 1-year local recurrence and overall intrahepatic recurrence rates were $19 \%$ and $45 \%$, respectively. Cheng et al $^{16)}$ reported randomized controlled trial conducted among 291 consecutive patients with HCC larger than $3 \mathrm{~cm}$ at a single center in China. During a median 28.5 months of follow-up, median survival times were 24 months in the TACE group (3.4 courses), 22 months in the RFA group (3.6 courses), and 37 months in the TACE-RFA group (4.4 courses). TACE-RFA was superior to TACE alone or RFA alone in improving survival for patients with HCC larger than $3 \mathrm{~cm}$.

Obviously, TACE is an acceptable way to decrease 


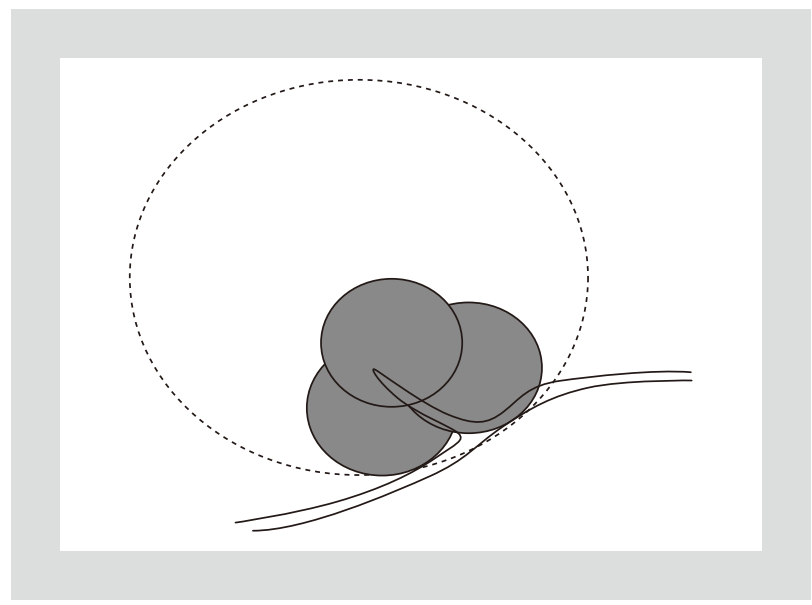

Figure 2 Computer representations of percutaneous ablation for entrance area of tumor feeding artery

heat sink effect during ablation. However, some patients were not candidate for TACE due to recurrence after surgery or poor respond to multiple TACE. Our group reported a new method -- percutaneous ablation of tumor feeding artery to control blood flow and increase coagulation effect during $\mathrm{RF}^{17)^{18)}}$. Before RF, we used color ultrasound to detect the tumor blood supply and identify the entrance of feeding vessel. As the first step of the percutaneous RF treatment, 2-3 overlapping high energy ablating foci, 2-3 cm each in diameter, were used to ablate the entering site of the feeding vessel (Fig. 2). Color Doppler ultrasound was used to confirm the absence of feeding artery. Blocking the blood flow in the tumor is helpful in decreasing heat loss during RF, and therefore the outcome improves. In our study, percutaneous RF to block feeding vessels were performed in $>3 \mathrm{~cm}$ and hypervascular HCC. The tumor necrosis rate at 1 and 6 month after RF was $92.6 \%(25 / 27)$ and $85.2 \%(23 / 27)$ in experiment group and $65.2 \%(15 / 23)$ and $56.5 \%(13 / 23)$ in control group (p = $0.024, \mathrm{p}=0.030)^{19)}$.

\section{Combining adjuvant cytotoxic therapies}

Previously, local hyperthermia was used to increase the effectiveness of chemotherapy by increasing blood flow, membrane permeability, local drug uptake, and metabolism in solid tumors ${ }^{20)}{ }^{21)}$. In Goldberg prior work, they demonstrated that combined RF ablation with commercially available longcirculating polyethylene glycol-coated liposomal doxorubicin preparation increases the extent of local tumor destruction and endpoint survival when compared with either RF ablation or intravenous doxorubicin alone in an animal tumor model $^{22-24)}$. In a randomized pilot clinical study, Goldberg et al administered liposomal doxorubicin 24 hours before RF ablation in 10 patients with 18 intrahepatic lesions ${ }^{23)}$. With this combination of RF ablation with adjuvant liposomal doxorubicin therapy, the authors were able to achieve increases of $25 \%-30 \%$ in coagulation volume in all tumors that received combination therapy compared with RF ablation alone.

Recently, Lencioni $\mathrm{R}$ et al reported their clinical trail results in HCC with doxorubicin-eluting bead (DEB)-enhanced $\mathrm{RF}^{24)}$. Twenty patients with single $\mathrm{HCC}$ ranging 3.3-7.0 cm (mean, $5.0 \mathrm{~cm} \pm 1.4$ ) showing evidence of residual viable tumour after standard RF ablation underwent intraarterial DEB administration (50-125 mg doxorubicin; mean, $60.2 \mathrm{mg} \pm 21.8$ ). The volume of treatment-induced necrosis-as measured on imaging--increased from $48.1 \mathrm{~cm}^{3} \pm 35.7$ after $\mathrm{RF}$ ablation to $75.5 \mathrm{~cm} \pm 52.4$ after DEB administration, with an increase of $60.9 \% \pm 39.0$. The enhanced effect resulted in confirmed complete response (CR) of the target lesion in 12 (60\%) of 20 patients. This result indicated DEB-enhanced RF ablation is safe and results in a high rate of CR in patients refractory to standard RF treatment.

Efficacy and complications in clinical application

The problems with ablating a large tumor are not trivial. Livraghi et $a l^{25)}$ described their experience treating 126 primary liver lesions $3.1 \mathrm{~cm}$ in diameter or larger (mean diameter, $5.4 \mathrm{~cm}$ ) in 114 consecutive patients. On the basis of the CT results obtained at least 5 months after treatment, they reported achieving complete tumor necrosis in 60 (47.6\%) of 126 lesions. Two major complications (death, hemorrhage requiring laparotomy) and 5 minor complications were observed. The single death was due to a break in sterile technique rather than to the RF procedure itself. Poon et $a l^{26)}$ has evaluated the safety and efficacy of RFA in 35 patients with medium and large HCC of $3-8 \mathrm{~cm}$ in diameter. There were no 
significant differences in the complication rate (17\% vs. $12 \%)$, treatment mortality rate (3\% vs. $0 \%)$, and complete tumor ablation rate (91\% vs. $94 \%)$ between medium and large $\mathrm{HCC}$, as well as small $\mathrm{HCC}(<3 \mathrm{~cm}$ in diameter). In another study by Bowles et $\mathrm{l}^{27)}, 76$ patients with locally advanced liver tumors (39 had colorectal liver metastases and 25 had HCC) underwent RFA. The mean tumor size was $3 \mathrm{~cm}$, ranging from 0.4 to $18 \mathrm{~cm}$. Local recurrence was detected in $9 \%$ of the patients at a mean follow-up of 15 months.

In our early study, we treated 121 hepatic tumors (74 HCC and 47 metastasis tumors; mean diameter, $4.75 \mathrm{~cm} \pm$ 0.93) with mathematical protocol. According to the CT results obtained 1 month after the treatment, the ablation was successful in $106(87.6 \%)$ of the 121 tumors. During the followup period of 3-26 months, the local recurrence rate was $24.0 \%$ $\left(29\right.$ of 121 tumors $^{9)}$. In our recent report ${ }^{28)}, 172$ patients who had $176>3.5 \mathrm{~cm}$ HCC and were not candidate for surgery or TACE were enrolled in this study. Established strategy for $>3.5 \mathrm{~cm}$ tumors included (1) multiple overlapping ablations based on mathematical protocol; (2) color US guided percutaneous ablation of tumor feeding artery (PAA) + RF for HCC with rich supply; (3) percuteneous arterial embolization $+\mathrm{RF}$ for HCC with high velocity flow. The ablation success rate was $91.5 \%$ (161/176 tumors) for all patients, 93.5\% (101/108 tumors) for $3.6-5.0 \mathrm{~cm} \mathrm{HCC}$ and $88.2 \%$ (60/68 tumors) for 5.1-7.0 cm HCC, respectively. Follow-up period ranged from 3 to 86 months with average 26 months. The local recurrence rate was $17.6 \%$ (31/176 tumors). The 1-, 3-, 5- overall survival were $80.4 \%, 52.0 \%$ and $37.5 \%$ for all patients, were $81.5 \%$, $57.5 \%$ and $46.5 \%$ for $3.6-5.0 \mathrm{~cm}$ HCC patients and were $80.1 \%$, $43.9 \%$ and $25.4 \%$ for $5.1-7.0 \mathrm{~cm}$ HCC patients, respectively (p $=0.174)$. The incidence of major complications was $3.5 \%$ $(6 / 172$ cases), including intraperitoneal hemorrhage $(n=2)$, hemothorax $(n=1)$, bowel perforation $(n=1)$ and needle tract seeding $(n=2)$. Of these, only one patient suffered from colon perforation 1 week after RFA treatment required surgical intervention.

\section{Summary}

The various techniques and protocols for large liver tumor have different advantages and disadvantages, but increased tumor destruction and long-term survivals have been reported. These results aslo add to the growing body of literature that suggests percutenous Radiofrequency (RF) ablation is a safe and effective method for the treatment of focal hepatic neoplasms. However, the management of large HCC is challenging, further trials are needed to confirm the clinical value of image-guided ablation in large hepatocellular HCC and more attempts are need to explore the outcome of combined RF with adjuvant therapy.

\section{References}

1) Yan K, Chen MH, Yang W, Wang YB, Gao W, Hao CY, Xing BC, Huang XF: Radiofrequency ablation of hepatocellular carcinoma: long-term outcome and prognostic factors. Eur J of Radiol 67: 336-347, 2008

2) Lencioni R, Cioni D, Crocetti L, Franchini C, Pina CD, Lera J, Bartolozzi C: Early-stage hepatocellular carcinoma in patients with cirrhosis: long-term results of percutaneous image-guided radiofrequency ablation. Radiology 234: 961-967, 2005

3) Abdalla EK, Vauthey JN, Ellis LM, Ellis V, Pollock R, Broglio KR, Hess K, Curley SA: Recurrence and outcomes following hepatic resection, radiofrequency ablation, and combined resection/ablation for colorectal liver metastases. Ann Surg 239: 818-825, 2004

4) Wood TF, Rose DM, Chung M, Allegra DP, Foshag LJ, Bilchik AJ: Radiofrequency ablation of 231 unresectable hepatic tumors: indications, limitations, and complications. Ann Surg Oncol 7: 593-600, 2000

5) Lencioni RA, Allgaier HP, Cioni D, Olschewski M, Deibert P, Crocetti L, Frings H, Laubenberger J, Zuber I, Blum HE, Bartolozzi C: Small hepatocellular carcinoma in cirrhosis: randomized comparison of radio-frequency thermal ablation versus percutaneous ethanol injection. Radiology 228: 235-240, 2003

6) Lam CM, Ng KK, Poon RT, Ai V, Yuen J, Fan ST: Impact of radiofrequency ablation on the management of patients with hepatocellular carcinoma in a specialized centre. Br J Surg 91: 334-338, 2004

7) Dodd GD 3rd, Frank MS, Aribandi M, Chopra S, Chintapalli KN: Radiofrequency thermal ablation: computer analysis of the size of the thermal injury created by overlapping ablations. AJR Am J Roentgenol 177: 777-782, 2001

8) Chen MH, Yang W, Yan K, et al: Large liver tumors: protocol for radiofrequency ablation and its clinical application in 110 patients-mathematic model, overlapping mode, and electrode placement process. Radiology 232: 260-271, 2004

9) Solbiati L, Ierace T, Tonolini M, Cova L: Guidance and monitoring of radiofrequency liver tumor ablation with contrast-enhanced ultrasound. Eur J Radiol 51 (Suppl): S19-23, 2004 
10) Chen MH, Yang W, Yan K, Dai Y, Wu W, Fan ZH, Callstrom MR, Charboneau JW: The role of contrast-enhanced ultrasound in planning treatment protocols for hepatocellular carcinoma before radiofrequency ablation. Clin Radiol 62: 752-760, 2007

11) Chinn SB, Lee FT Jr, Kennedy GD, Chinn C, Johnson CD, Winter TC 3rd, Warner TF, Mahvi DM: Effect of vascular occlusion on radiofrequency ablation of the liver: results in a porcine model. AJR Am J Roentgenol 176: 789-795, 2001

12) Yang W, Chen MH, Wang MQ, Cui M, Gao W, Wu W, Wu JY, Dai Y, Yan K: Combination therapy of radiofrequency ablation and transarterial chemoembolization in recurrent hepatocellular carcinoma after hepatectomy compared with single treatment. Hepatol Res 39: 231-240, 2009

13) Goldberg SN, Hahn PF, Halpern EF, Fogle RM, Gazelle GS: Radio-frequency tissue ablation: effect of pharmacologic modulation of blood flow on coagulation diameter. Radiology 209: 761-767, 1998

14) Buscarini L, Buscarini E, Di Stasi M, Quaretti P, Zangrandi A: Percutaneous radiofrequency thermal ablation combined with transcatheter arterial embolization in the treatment of large hepatocellular carcinoma. Ultraschall Med 20: 47-53, 1999

15) Rossi S, Garbagnati F, Lencioni R, Allgaier HP, Marchianò A, Fornari F, Quaretti P, Tolla GD, Ambrosi C, Mazzaferro V, Blum HE, Bartolozzi C: Percutaneous radio-frequency thermal ablation of nonresectable hepatocellular carcinoma after occlusion of tumor blood supply. Radiology 217: 119-126, 2000

16) Cheng BQ, Jia CQ, Liu CT, Fan W, Wang QL, Zhang ZL, Yi CH: Chemoembolization combined with radiofrequency ablation for patients with hepatocellular carcinoma larger than $3 \mathrm{~cm}$ : a randomized controlled trial. JAMA 299: 1669-1677, 2008

17) Chen MH, Wei Y, Yan K, Gao W, Dai Y, Huo L, Yin SS, Zhang H, Poon RT: Treatment strategy to optimize radiofrequency ablation for liver malignancies. J Vasc Interv Radiol 17: 671-683, 2006

18) Chen MH, Yang W, Yan K, Hou YB, Dai Y, Gao W, Zhang H, Wu W: Radiofrequency ablation of problematically located hepatocellular carcinoma: tailored approach. Abdom Imaging 33: 428-436, 2008

19) Hou YB, Chen MH, Yan K, Wu JY, Zhang H, Yang W, Wu W: Feasibility of improving radiofrequency ablation of hepatocellular carcinoma by percutaneously blocking tumor-feeding vessels. Zhongguo Yi Xue Ke Xue Yuan Xue Bao 30: 448-454, 2008

20) Hahn GM: Potential for therapy of drugs and hyperthermia. Cancer Res 39: 2264-2268, 1979

21) Wallah DFG: Basic mechanism in tumor thermotherapy. J Mol Med 17: 381-403, 1977

22) Monsky WL, Kruskal JB, Lukyanov AN, Girnun GD, Ahmed M, Gazelle GS, Huertas JC, Stuart KE, Torchilin VP, Goldberg SN: Radio-frequency ablation increases intratumoral liposomal doxorubicin accumulation in a rat breast tumor model. Radiology 224: 823-829, 2002

23) Goldberg SN, Kamel IR, Kruskal JB, Reynolds K, Monsky WL, Stuart KE, Ahmed M, Raptopoulos V: Radiofrequency ablation of hepatic tumors: increased tumor destruction with adjuvant liposomal doxorubicin therapy. AJR Am J Roentgenol 179: 93-101, 2002

24) Lencioni R, Crocetti L, Petruzzi P, Vignali C, Bozzi E, Della Pina C, Bargellini I, Cioni D, Oliveri F, De Simone P, Bartolozzi C, Brunetto M, Filipponi F: Doxorubicin-eluting bead-enhanced radiofrequency ablation of hepatocellular carcinoma: a pilot clinical study. J Hepatol 49: 217-222, 2008

25) Livraghi T, Goldberg SN, Lazzaroni S, Meloni F, Ierace T, Solbiati L, Gazelle GS: Hepatocellular carcinoma: radio-frequency ablation of medium and large lesions. Radiology 214: 761-768, 2000

26) Poon RT, Ng KK, Lam CM, Ai V, Yuen J, Fan ST: Effectiveness of radiofrequency ablation for hepatocellular carcinomas larger than 3 cm in diameter. Arch Surg 139: 281-287, 2004

27) Bowles BJ, Machi J, Limm WM, Severino R, Oishi AJ, Furumoto NL, Wong LL, Oishi RH: Safety and efficacy of radiofrequency thermal ablation in advanced liver tumors. Arch Surg 136: 864-869, 2001

28) Chen MH, Yang W, Wu W, et al: Treatment strategy and efficacy of radiofrequency ablation in 172 patients with $>3.5 \mathrm{~cm}$ hepatocellular carcinoma. Radiological Society of North America Scientific Assembly and Annual Meeting Program 120: 553, 2008 